and subsequent fostering the diarrhoea ceased and the child's weight gain was dramatic (figure).

\section{Discussion}

The deliberate administration of harmful substances was included in early descriptions of child abuse. ${ }^{1}$ These substances include both household products and drugs, which were often prescribed for adults in the family. ${ }^{2}$ Previous reports have emphasised that a prerequisite for diagnosis is a low threshold of suspicion. ${ }^{34}$ Disturbances of bowel function with poor weight gain are commonly seen in infants, but reports of deliberate overdosing with dangerous drugs are relatively uncommon, and laxative abuse in very young children is exceedingly rare. These factors, the exemplary behaviour of the parents, and the trust which doctors place in parental histories, militate against early diagnosis with potentially life threatening consequences.

Even when the diagnosis is considered, proving it is often difficult and in this case the stool electrolytes were vital in distinguishing an osmotic from a secretory diarrhoea. The severity of weight loss and the near fatal episode of dehydration emphasises the need to consider pharmacological abuse where the clinical picture is unusual. ${ }^{2}$

\section{References}

' Kempe CH, Silverman FN, Steele BF, Droegemueller W, Silver HK. The battered child syndrome. JAMA 1962;181:17-24.

${ }^{2}$ Rogers D, Tripp J, Bentovin A, Robinson A, Berry D, Goulding R. Non-accidental poisoning: an extended syndrome of child abuse. Br Med J 1976;i:793-6.

${ }^{3}$ Meadow R. Munchausen syndrome by proxy. The hinterland of child abuse. Lancet 1977;ii:343-5.

${ }^{4}$ Watson JBG, Davies JM, Hunter JLP. Non-accidental poisoning in childhood. Arch Dis Child 1979;54:143-4.

Correspondence to Dr MS Tanner, Department of Child Health, Clinical Sciences Building, Leicester Royal Infirmary, PO Box 65, Leicester LE2 7LX.

Accepted 7 March 1988

\title{
Clostridium difficile in an oncology unit
}

\section{A L BRUNETTO, ${ }^{*}$ A D J PEARSON,* A W CRAFT,* AND S J PEDLER $\dagger$ \\ *Department of Child Health, The Medical School, University of Newcastle upon Tyne, and †Department of Clinical Microbiology, Royal Victoria Infirmary, Newcastle upon Tyne}

SUMmaRY In one year 21 new cases of Clostridium difficile infection occurred on a paediatric oncology unit. Eleven cases were in a two month period. This infection should be regarded as a communicable disease. Investigations to detect $C$ difficile should be carried out in children with malignant disease who have diarrhoea.

Clostridium difficile has been increasingly recognised as an aetiological agent of acute and chronic antibiotic associated diarrhoea and pseudomembranous colitis. ${ }^{1}$ There have been reports of outbreaks in hospital units as well as sporadic cases. Patients with malignant disease receiving immunosuppressive treatment are at risk of opportunistic infection. As they also receive numerous courses of antibiotics and have frequent hospital admissions throughout their treatment they are at particular risk of $C$ difficile infection. ${ }^{2}{ }^{3}$ In addition cancer chemotherapy may predispose to gut colonisation with $C$ difficile. ${ }^{3}$ We report an outbreak of infection with this organism in a paediatric oncology unit associated with a change in antibiotic policy.

\section{Patients and methods}

All patients admitted to the paediatric oncology unit, a 10 bedded ward exclusively for children with cancer, in Newcastle upon Tyne during 1986 who had diarrhoea associated with $C$ difficile were included in this study. Diarrhoea was defined as a change from normal bowel pattern with passage of two or more consecutive unformed stools. Stool specimens from patients with diarrhoea were examined for the presence of $C$ difficile and its cytotoxin in addition to routine virological and bacteriological investigations.

Specimens were inoculated onto Columbia blood agar containing cycloserine and cefoxitin as selective agents. Detection of cytotoxin was performed by standard laboratory methods. ${ }^{4}$

Since 1981 patients on the unit with neutropenia and fever have received empirically gentamicin and azlocillin. During 1985 only $61 \%$ of Gram negative bacilli isolated were sensitive to azlocillin, therefore in March 1986 cefuroxime was substituted for azlocillin.

Because of an apparent increase in the number of patients with $C$ difficile infection early in 1986, stool 
specimens were examined for $C$ difficile from all children admitted during the five month period from the middle of May to October 1986. Also the use of cefuroxime was discontinued and azlocillin and gentamicin were instituted.

All patients who were positive for culture or toxin for $C$ difficile received an oral course of vancomycin (125 mg orally three times daily) and if the culture or toxin remained positive a further course was given consisting of three days of vancomycin with a three day rest period repeated three times. ${ }^{5}$

\section{Results}

During the year 21 patients, nine girls and 12 boys, age 1-19 years had one or more episodes of diarrhoea associated with $C$ difficile during some stage of their treatment (figure). Eleven of these occurred in a two month period. Nineteen had a mild illness but in two the symptoms were more serious with bloody diarrhoea and abdominal pain. All were receiving chemotherapy for either leukaemia (11 patients) or solid tumours (10 patients). At the time of presentation with $C$ difficile associated diarrhoea, 10 patients had an absolute neutrophil count less than $1.0 \times 10^{9} / 1$. In 15 patients both the organism and the toxin were present in faeces but in five only the organism and in one only the toxin was detected.

In all 21 patients symptoms resolved with treatment with oral vancomycin and the organism and its toxin could not be detected in stools after treatment. In nine patients $(43 \%)$, however, $C$ difficile or its toxin were detected again at a later date. Such recurrence of colonisation was usually, but not always, associated with symptoms. Some children

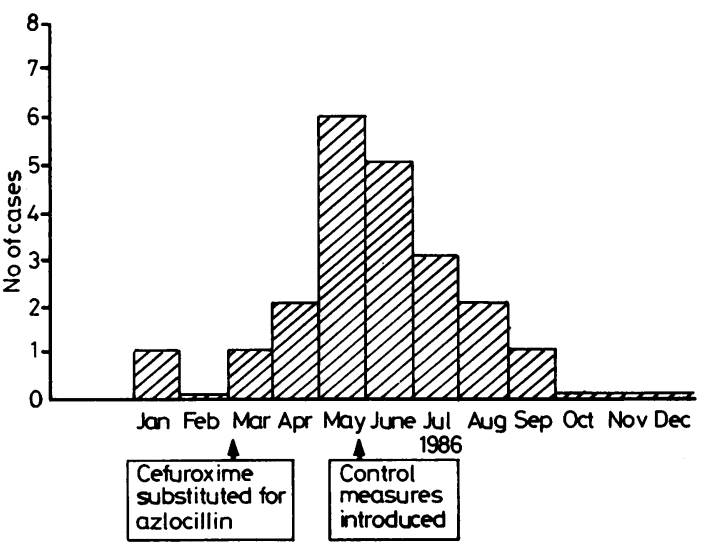

Figure Number of new cases of Clostridium difficile associated diarrhoea in each month for 1986.
Table Recurrence of clostridium difficile in the stool of 26 patients

\begin{tabular}{llll}
\hline $\begin{array}{l}\text { lsolation of } \\
\text { Clostridium difficile }\end{array}$ & \multicolumn{2}{c}{ Diarrhoea } \\
$\begin{array}{l}\text { Noof } \\
\text { children } \begin{array}{l}\text { Total No } \\
\text { of episodes }\end{array}\end{array}$ & \\
\hline
\end{tabular}

\begin{tabular}{lrrrr}
\hline $\begin{array}{l}\text { Symptomatic patients } \\
\text { No of relapses }\end{array}$ & & & \\
0 & 12 & 12 & 12 & 0 \\
1 & 5 & 10 & 8 & 2 \\
2 & 3 & 9 & 7 & 2 \\
3 & 1 & 4 & 4 & 0 \\
Subtotal & 21 & 35 & 31 & 4 \\
$\begin{array}{l}\text { Asymptomatic patients } \\
\text { No of relapses }\end{array}$ & & & \\
0 & 3 & 3 & 0 & 3 \\
1 & 2 & 4 & 0 & 4 \\
Subtotal & 5 & 7 & 0 & 7 \\
\hline Total & 26 & 42 & 31 & 11 \\
\hline
\end{tabular}

had multiple recurrence, giving a total of 35 episodes of infection in 21 patients (table).

During the five month period in which routine screening of all patients'admitted to the ward was carried out, 77 patients were admitted on 272 occasions. In addition to the 14 symptomatic patients during this screening period described above, five asymptomatic patients were found to have $C$ difficile in their stools. In two of these patients colonisation recurred after treatment (table).

Toxin was detected in 18 out of $31(58 \%)$ episodes of $C$ difficile infection associated with diarrhoea but only in two of 11 asymptomatic isolations $(\mathrm{p}<0.02)$. All 10 episodes of $C$ difficile infection in patients with an absolute neutrophil count of less than $1.0 \times 10^{9} / 1$ were symptomatic.

\section{Discussion}

C difficile has been isolated occasionally in the paediatric oncology unit in Newcastle since 1980. The outbreak described above was associated with a change in antibiotic policy from the use of azlocillin to cefuroxime. Although $C$ difficile associated diarrhoea does occur without prior exposure to antibiotics, most cases reported do implicate an association and the cephalosporins appear to be a particular problem. ${ }^{6}$

There are a number of potential causes of diarrhoea in children receiving treatment for malignant disease. In addition to bacterial, viral, and parasitic infection chemotherapy can cause small 
bowel mucosal damage resulting in diarrhoea. It is important that diarrhoea is not accepted as a normal symptom of cancer chemotherapy and that stool specimens are sent for full bacteriological and viral investigations including examination for the presence of $C$ difficile and its toxin. Relapse after the discontinuation of treatment with vancomycin, metronidazole, or bacitracin has been reported with incidences ranging from $5 \%$ to $59 \%$. $^{5}$ In the present series, the symptomatic relapse rate was $38 \%$.

There now seems little doubt that $C$ difficile infection is a communicable disease, which may cause outbreaks of infection in hospital. ${ }^{2}{ }^{3}$ Patients with diarrhoea known or suspected to be due to $C$ difficile should be isolated and 'barrier-nursed' in the same way as patients with other recognised gastrointestinal pathogens. The low incidence of asymptomatic carriage detection in this study suggests that screening is probably not worthwhile as a routine preventive measure except during an outbreak. Antibiotic policies should be reviewed if an outbreak occurs.

It is possible that the outbreak could have been aborted if control measures had been commenced sooner. Recognition that an outbreak is occurring is therefore vital, particularly in units which already have a low 'background' incidence of infection.

Therefore we suggest that paediatric oncology units should monitor the number of cases of $C$ difficile infection and if there is any evidence of an increasing incidence control measures should be started.
With early treatment severe life threatening illness may be prevented. The presence of either the toxin or the organism may be related to symptoms, and the absence of the toxin should not dissuade the physician from considering $C$ difficile as a pathogen and commencing specific treatment.

We thank CAPES (Ministry of Education of Brazil) for the support of ALB, the North of England Children's Cancer Research Fund, and Dr MM Reid for critical review of the manuscript.

\section{References}

${ }^{1}$ Bartlett JG, Chang TW, Gunwith M, Gorback SL, Onderdunk AB. Antibiotic associated pseudomembranous colitis due to toxin producing clostridia. $N$ Engl J Med 1978;298:531-4.

2 Morris JG, Jarvis WR, Nunez-Montiel OL, et al. Clostridium difficile. Colonization and toxin production in a cohort of patients with malignant haematological disorders. Arch Intern Med 1984;144:967-9.

${ }^{3}$ Cudmore MA, Silva J, Fekety R, Liepman MK, Kim KH Clostridium difficile colitis associated with cancer chemotherapy. Arch Intern Med 1982;142:333-5.

${ }^{4}$ Burden DW. Laboratory investigations of antibiotic-associated diarrhoea. London: Association of Clinical Pathologists: Broadsheet 102, June, 1982.

5 Bartlett JG. Treatment of Clostridium difficile colitis. Gastroenterology 1985;89:1192-5.

${ }^{6}$ Bartlett JG. Antimicrobial agents implicated in Clostridium difficile associated diarrhoea and colitis. Johns Hopkins Medical Journal 1981;6:6-9.

Correspondence to Dr AL Brunetto, Department of Child Health, University of Newcastle upon Tyne, The Medical School, Framlington Place, Newcastle upon Tyne, NE2 $4 \mathrm{HH}$.

Accepted 5 April 1988
SUMmary Daily growth of the lower leg in a child receiving alternate day oral steroids for Crohn's disease was measured by knemometry. Growth occurred on days free of treatment. This may represent a direct observation of the growth sparing effect of alternate day steroid medication.

The knemometer is designed to measure changes in tibial length with great accuracy. ${ }^{1}$ The technique has recently been rigorously assessed and its power in detecting changes in growth over short (less than eight weeks) periods confirmed. ${ }^{2}$ The definition of measurement is $0.1 \mathrm{~mm}$ and the coefficient of variation of six readings by one observer is $0.09 \%$ giving a precision (coefficient of variation $\times$ mean value of tibial length) of $0.2 \mathrm{~mm}$. Intradaily variation in tibial length has been described in normal children, ${ }^{3}$ and day to day fluctuations in growth shown in children receiving growth hormone using the same technique. ${ }^{4}$

The recent suggestion that this technique may be used as a sensitive marker of growth supression prompted us to measure the daily lower leg length of a child receiving alternate day oral steroids for the treatment of Crohn's disease. ${ }^{5}$ 